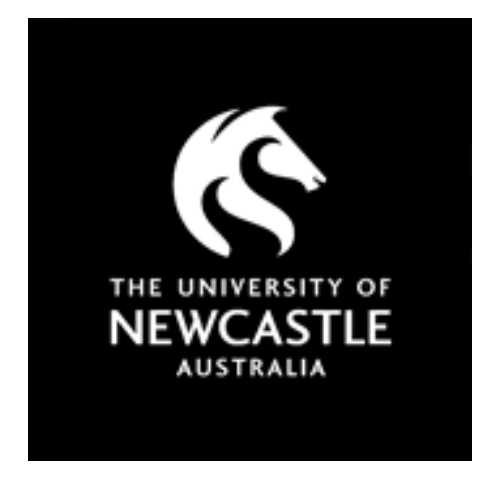

NOVA

University of Newcastle Research Online

nova.newcastle.edu.au

Lee, Ju Hyan; Gu, Ning; Jupp, Julie; Sherratt, Sue 'Evaluating creativity in parametric design processes and products: a pilot study', 5th International Conference on Design Computing and Cognition (DCC'12). Design Computing and Cognition '12 (College Station, TX 7-9 June, 2012) p. 165-183 (2014)

Available from: http://dx.doi.org/10.1007/978-94-017-9112-0 10

The final publication is available at www.springerlink.com

Accessed from: $\underline{\text { http://hdl.handle.net/1959.13/1302389 }}$ 


\title{
Evaluating Creativity in Parametric Design Processes and Products: A Pilot Study
}

\author{
Ju Hyun Lee ${ }^{1}$, Ning Gu ${ }^{1}$, Julie Jupp ${ }^{2}$, and Sue Sherratt ${ }^{1}$ \\ ${ }^{1}$ University of Newcastle; ${ }^{2}$ University of Technology Sydney, Australia
}

Parametric design is an emerging research issue in the design domain. However, our current understanding of creativity in relation to either a process or product standpoint is limited. This paper presents a formal approach for the description and identification of creativity from both perspectives. The framework combines: (i) protocol analysis for encoding cognitive design activities, providing a process-based evaluation of creativity, and (ii) consensual assessment of parametric products, providing a product-based evaluation of creativity. The coding scheme is based on the creative acts: Representation, Perception, and Searching for a Solution. The consensual assessment technique is based on a series of creativity evaluations undertaken by an expert panel. The effectiveness of this approach was examined in a pilot study. Findings show the capture of cognitive activities and identification of creative patterns, revealing how they correspond to the creativity levels of parametric design products. The results identify conditions that have the potential to enhance creativity in parametric design. This research provides a promising procedure not yet available and contributes to the development and verification of a formal approach for evaluating creativity in parametric design.

\section{Introduction}

Parametric design has become an increasingly popular approach to Computer-aided Design (CAD), resulting in the emergence of a global architectural style, known as 'parametricism' [1]. The popularity of this approach is due, on the one hand, to the generation of unusual forms, often with complex geometries and increased technical sophistication; on the 
other hand, by virtue of the recognition of these designs being novel, useful and, arguably, creative - important goals in non-routine design.

Whilst a profusion of parametric design solutions have been constructed and widely illustrated in architectural magazines, studies of parametric design activities and outputs is relatively nascent. Consequently, our understanding of the generative and evolutionary aspects of parametric design and the role of creativity - from either a process or product standpoint -is limited.

Our investigation explores this knowledge gap. This paper presents a formal evaluation framework developed by the authors for assessing creativity in parametric design. This framework is based on a two-pronged approach: (1) a protocol analysis procedure - providing a process-based evaluation of creativity, and (2) a selective criteria-based assessment method, providing a product-based evaluation of creativity. The remainder of this paper is divided into four parts. Section 2 explores the related literature. Section 3 then presents a conceptual framework for evaluating creativity in parametric design. A pilot study illustrating the research approach and framework is presented in Section 4, detailing its implementation and resulting empirical data. Finally, Section 5 concludes with a discussion of the empirical evidence and outlines the directions for future work.

\section{Background}

Creativity has been described in terms such as "creative thinking", "problem solving", "imagination", or "innovation" [2]. Design activities involve problem solving [3], which could be characterized as a cognitive process [4]. Creativity should be a natural component of the design process [5]. In the design domain, process and cognition are core themes [6], and much of the related research has aimed to enhance design creativity. Researchers [5, 7-9] have studied designers' sketching activities in attempts to understand the creative process in the early conceptual design stages, revealing that sketching activities are linked in various ways to creativity. In other approaches, researchers have compared sketching activities with those used in the application of traditional CAD tools [4, 10]. These studies show that sketching, with its flexible and intuitive characteristics, has advantages over CAD tools in terms of creativity. Ibrahim and Rahimian [11] illustrate through their studies that both sketching and conventional CAD tools have limitations in supporting conceptual design. Sketching has limitations in constructing complex 
designs. Conventional CAD tools can hinder creativity due to their inflexibility.

Design studies indicate that sketching enhances creativity through reconstructing $[4,10,12]$ and regulating [8] mental imagery. Thus, in order for digital design techniques to become more flexible and intuitive, they should provide interactive imagery so as to enable the designer to perceive visuo-spatial features and organizational relations, and generate alternative solutions [4]. Such techniques need to also consider the management of part-whole relationships, design hierarchies, topologygeometry relationships, structuring of ill-structured problems, and restructuring of problem parameters [8]. Research has shown these characteristics are evident in parametric design $[1,13,14]$. Advances in such digital design techniques are providing an increasing capacity to encode and evaluate generative processes that can intuitively support design exploration whilst maintaining flexibility. With the complexity and power of contemporary parametric design software, the impact of such tools on creativity in the conceptual design phases remains relatively unexplored. However, recent research has illustrated that parametric design can support creativity $[15,16]$, with some parametric design tools providing intuitive restructuring and flexible regulating environments [15, $16]$, thereby allowing interactions that can engage complexity $[13,14]$.

Lee et al. [2] explained that parametric design is related to both divergent and convergent thinking, two of the most important processes in models of creativity. Divergent thinking is connected to the parameters and generative rules available in parametric design environments, while convergent thinking comprises the rules which define constraints for the most correct (or satisfactory) answer to a design question. Iordanova, et al. [15] argue that generative modeling using parametric design tools can contribute to creativity. Chusllp and Jin [17] proposed a cognitive activity model consisting of three loops for problem redefinition, idea stimulation, and concept reuse. Generative parametric-based design solutions are evolved through extensive iteration and regeneration by modified parameters and rules (algorithms). Scripting or coding activities are regarded as a channel for creativity and a means of representing design ideas [16]. Thus, whilst generative parametric modeling presents a different context for design during the conceptual stages than that of sketching, divergent and convergent thinking is maintained. From this perspective, parametric design is capable of supporting not only the generation of ideas but also their evolution, leading to creativity. This paper therefore focuses on the interactive and algorithmic activities of parametric design that are significant in the creative process which underlie the generation of creative outcomes. 


\section{Framework for Evaluating Creativity in Parametric Design}

The framework for evaluating creativity in parametric design is based on the key work of Rhodes [18]. Rhodes aimed to deal with both a cognitive approach to the design process and a confluence approach to design products. This approach [18] classifies strands of creativity into four perspectives - known as the four Ps of creativity: person, process, press (environment), and product. This conceptualization of creativity recognizes that design environments are influenced by physical and social contexts that can affect the creative design process, the quality of the design product, and levels of personal creativity. However, it is difficult to evaluate and investigate all design environments within a single research framework. Simonto [19] suggests that all three perspectives except for 'press' should be integrated into a unified view of scientific creativity. Although Hasirci and Demirkan [5] present a framework derived from Rhodes' four Ps, they also paid attention to the relationships among the three essential components of creativity (still excluding 'press'). They claimed that person and product follow process. Design process must therefore be a core theme in the study of creativity as it can reveal the characteristics of creativity. This approach is significant to the study of creativity in parametric design.

Figure 1 illustrates our proposed framework for evaluating creativity in parametric design. Taking into account Rhodes' four P's, the framework will be applied to designing (process) and design (product) in parametric design environments (press), whilst also accounting for design strategies and preferences as part of Rhodes' personal creativity (person). The framework is divided into two components, viz. design process and design product. The evaluation of the creative design process uses protocol analysis and the evaluation of the design outcomes adopts an expert assessment approach based on the Consensual Assessment Technique (CAT) [20].

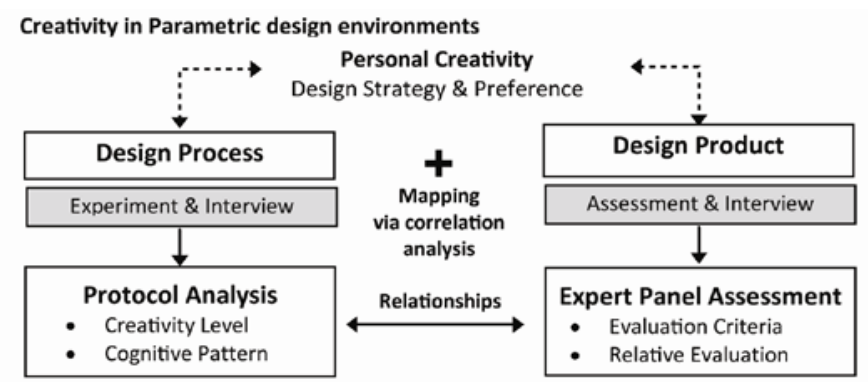

Fig 1. A framework for evaluating creativity in parametric design 
This research argues that by encoding segments of the design process in sequence and using the coding scheme developed here, cognitive patterns can be identified which will provide an evaluation of creativity in parametric designing. Furthermore, by mapping these results with the creativity assessments provided by an expert panel, it will be possible to enhance our understanding of process-based creativity relative to productbased creativity. Hence, it was important that the proposed coding scheme be capable of describing levels of creativity and identifying cognitive patterns throughout the creative process.

\subsection{Coding Scheme and Protocol Analysis}

To evaluate creativity in the parametric design process, we have adopted protocol analysis [21-23] which has been widely used to explore cognitive activities in the design process. In protocol analysis, a coding scheme for describing activities of creativity is essential and should be devised to be suitable for the purpose and the design environments. Therefore, for effective analysis, a coding scheme should be specifically developed to suit the process of parametric design.

The two coding schemes developed by Suwa et al. [23] and Gero \& Neill [24] have been widely used in the design domain. The former targets the content-oriented aspects of designing, whilst the latter targets the process-oriented aspects of designing [25]. For this research, the code developed by Suwa et al. [23] is suitable for examining the representation and perception aspects in parametric designing. Similarly, the code developed by Gero \& Neill [24] is valuable for studying aspects of searching for solutions in parametric designing. The coding scheme for analyzing how parametric design process supports creativity (see Table 1) is based on the adaptation from these two influential coding schemes.

For our coding scheme, three levels of cognitive processes identifying creativity in the design process are defined: 'Representation', 'Perception', and 'Searching for a Solution'. These levels are informed by the cognitive processes in creativity identified by Hayes [26]. To generate a cognitive pattern suitable to the conditions of parametric design, each level is then separated into two subcategories: (1) Geometry, and (2) Algorithm, whilst 'Searching for a Solution' comprises three subcategories, viz.: Finding Idea, Evaluation, and Adopting Idea. Subclasses were then defined for detailed actions based on both design [27] and cognitive actions [22]. 
Table 1 Coding scheme for exploring the parametric design process

\begin{tabular}{|c|c|c|c|}
\hline $\begin{array}{l}\text { Creativity lev- } \\
\text { el }\end{array}$ & Category & Subclasses & Description \\
\hline \multirow[t]{3}{*}{ Representation } & Geometry & $\begin{array}{l}\text { RG-Geometry } \\
\text { RG-Change }\end{array}$ & $\begin{array}{l}\text { create geometries without an al- } \\
\text { gorithm } \\
\text { change existing geometries }\end{array}$ \\
\hline & Algorithm & $\begin{array}{l}\text { RA-Parameter } \\
\text { RA-Change Parame- } \\
\text { ter } \\
\text { RA-Rule } \\
\text { RA-ChangeRule } \\
\text { RA-Reference }\end{array}$ & $\begin{array}{l}\text { create initial parameters } \\
\text { change existing parameters } \\
\text { create initial rules } \\
\text { change existing rules } \\
\text { retrieve or get references }\end{array}$ \\
\hline & & R-Generation & make generation (or variation) \\
\hline \multirow[t]{2}{*}{ Perception } & Geometry & PG-Geometry & attend to existing geometries \\
\hline & Algorithm & $\begin{array}{l}\text { PA-Algorithm } \\
\text { PA-Reference }\end{array}$ & \begin{tabular}{|l|} 
attend to existing algorithms \\
attend to existing references \\
\end{tabular} \\
\hline \multirow[t]{5}{*}{$\begin{array}{l}\text { Searching for } \\
\text { a Solution }\end{array}$} & $\begin{array}{l}\text { Finding } \\
\text { Idea } \\
\text { (Geometry) } \\
\text { (Algorithm) }\end{array}$ & $\begin{array}{l}\text { SF-Initial Goal } \\
\text { SF-Geometry Sub- } \\
\text { Goal } \\
\text { SF-Algorithm Sub- } \\
\text { Goal }\end{array}$ & $\begin{array}{l}\text { introduce new ideas (or goals) } \\
\text { based on a given design brief } \\
\text { introduce new geometric ideas } \\
\text { extended from a previous idea } \\
\text { introduce new algorithmic ideas } \\
\text { extended from a previous idea }\end{array}$ \\
\hline & \multirow[t]{2}{*}{\begin{tabular}{|l|} 
Evaluation \\
\end{tabular}} & SE-Geometry & $\begin{array}{l}\text { evaluate primitives or existing } \\
\text { geometries }\end{array}$ \\
\hline & & $\begin{array}{l}\text { SE-Parameter } \\
\text { SE-Rule } \\
\text { SE-Reference } \\
\end{array}$ & $\begin{array}{l}\text { evaluate existing parameters } \\
\text { evaluate existing rules } \\
\text { evaluate existing references }\end{array}$ \\
\hline & \multirow{2}{*}{$\begin{array}{l}\text { Adopting } \\
\text { Idea } \\
\text { (Algorithm) }\end{array}$} & SA-Geometry & adopt new ideas to geometries \\
\hline & & $\begin{array}{l}\text { SA-Parameter } \\
\text { SA-Rule } \\
\text { SA-Reference }\end{array}$ & $\begin{array}{l}\text { adopt new ideas to parameters } \\
\text { adopt new ideas to rules } \\
\text { adopt new ideas to retrieve or get } \\
\text { internal/external references }\end{array}$ \\
\hline
\end{tabular}

\subsection{Expert Panel Assessment}

The use of 'expert panel assessments' $[28,29]$ based on CAT $[9,16]$ is a valuable and often-used approach for measuring the creativity of design products. The CAT technique is based on the notion that a measure of the creativity of an artifact is the combined assessment of experts in that field. Unlike other measures of creativity, CAT is not based on any particular theory of creativity, which means that its validity (which has been well established empirically) is not dependent upon the validity of any particular theory of creativity [30]. In employing this technique, a basic requirement of an expert panel assessment is that its members are familiar with the design domain and the techniques required to produce the design $[28,31]$. 
From this standpoint, a series of assessment tasks were designed to evaluate the parametric design models, namely:

i. Independent non-criteria based assessment - using their own judgment of creativity, panel members must evaluate the creativity of each design model independently from the other models,

ii. Comparative non-criteria based assessment - using their own judgment of creativity, panel members must evaluate design models relative to one another.

iii. Criterion-based assessment - using specific evaluation criteria, the panel must evaluate each design model.

Evaluation criteria were selected by reviewing the literature on creativity assessment [20,32]. Criteria are often comprised of three categories: novelty, usefulness, and aesthetics. Based on these, a number of subscales can then be derived. For example, in the Creative Product Semantic Scale, or CPSS, Christiaans [29] used a large number (70 in total) of bipolar subscales. However, such a large number of criteria would be time-consuming and even confusing for assessors. The subscales in our research were selectively adopted from the CAT [20, 32] and CPSS methods [33]. Both instruments have been widely used in design creativity assessment. Table 2 shows the evaluation criteria selected here to assess the creativity of parametric design products.

Table 2 Criteria for assessing the creativity parametric design products

\begin{tabular}{|l|l|l|}
\hline Novelty & Usefulness & Aesthetics \\
\hline $\begin{array}{l}\text { Originality } \\
\text { Innovation }\end{array}$ & $\begin{array}{l}\text { Technical Quality } \\
\text { Functional Quality } \\
\text { Integration Capacity }\end{array}$ & $\begin{array}{l}\text { Attractiveness } \\
\text { Expressiveness } \\
\text { Complexity }\end{array}$ \\
\hline
\end{tabular}

The pilot study, presented in Section 4, utilizes all three assessment tasks and evaluation criteria, including Novelty, Usefulness and Aestheticbased Complexity. However the subscales shown in Table 2 were not tested. Whilst the subscales require further development, the pilot study focuses on the parametric design process in relation to evaluations of product-based creativity relative to the three main criteria mentioned above. 


\subsection{Mapping via Correlation Analysis}

Finally, the framework includes mapping the cognitive activities of the design process and the expert panel's assessment. Correlation analysis is used to identify particular process patterns in parametric designing that may enhance or hinder different aspects of creative design. Participants with similar results on the expert panel assessment are grouped together. Correlation analysis is then used to examine significant similarities or differences in the protocol analysis results within and across groups.

\section{Pilot Study}

The pilot study tests the two components of the formal framework and the correlation analysis mapping technique so as to assess their effectiveness. Thus, a parametric design experiment and an expert panel assessment were conducted.

\subsection{Process-based Evaluation of Creativity}

\section{Design Experiment Procedure}

A written design brief was given to participants and described verbally by the experimenter. The brief concerned the conceptual design of a high-rise building. This is a simple form generation design task, containing five specific design requirements, viz. that the high-rise building will (i) have two main functional areas of offices and a hotel; (ii) have a maximum floor area of 2,500 square meters ( $50 \mathrm{~m}$ x $50 \mathrm{~m}$ ) per floor; (iii) be over 40 stories high; (iv) reflect transformations of structural forces using external data (optional); and (v) be a designated regional landmark. Participants were instructed to "think-aloud" or provide a running commentary of their actions and thoughts.

Participants were given one hour to undertake the design task and were video-recorded utilizing parametric modeling tools of their choice, including, e.g., Rhino, Grasshopper, Maya, and Python scripting. Further, as parametric scripting could be very technical for designers and the time restriction could be a potential source of stress for participants and limit their designing, all participants were informed that they could continue if more time was required.. Design deliverables were specified in the brief, namely: (i) a 3D model and related files, and (ii) rendered or captured 
images to clearly represent the conceptual design solution and illustrate its main attributes.

After each design session, the students then participated in a recorded post experiment interview with researchers so as to report and explain their retrospective thoughts and activities in terms of their think-aloud protocols, whilst watching the recorded video. In addition, four specific questions to describe their experiences and preferences on creativity in parametric design environments were posed. Each student used a different parametric design environment: Grasshopper, Maya Script Editor (SE), and Python.

\section{Protocol Analysis Procedure}

The protocols were firstly divided into smaller segments. Suwa et al. [23] categorized methods of segmentation in two approaches, firstly, using pauses or syntactic markers, and the second based on the participant's intentions or the content of thoughts and activities. This study employs the latter technique and segmentation was undertaken by a single researcher. An intention (or content of thought and activities) represents a segment and it was encoded as at least one or as several of the subclasses described in Table 1. Furthermore, segmentation was based on the video recording of the computer screen. The protocols of each recorded video were directly transcribed using NVivo 9 research software and automatically segmented into smaller episodes. The coder encoded the transcriptions using the scheme presented in Section 3.1. The average value of the number of segments was 263.5 (Student A: 142, Student B: 286, Student C: 368). Over $90 \%$ of each protocol was encoded in our coding scheme.

The coded data and transcriptions were transferred to Excel 2007 spreadsheets to encode the data series again and inspect the codes themselves. The coded data were visualized in simple graphical forms to facilitate the exploration and identification of patterns and characteristics.

\section{Coding Results of the Parametric Design Process}

Table 3 shows the coding results describing the percentage of the frequency weighted by time span (calculated by time duration of each code). This allows us to figure out time usage in stages of design process [5] as well as main activities of each participant. On average, the coverage of 'Representation' accounts for $46.4 \%$, 'Perception' accounts for $22.0 \%$, and 'Searching for a Solution' accounts for $52.4 \%$. 'RA-Rule' and 'RAChangeRule' were dominant features in each student's parametric design process. There was little 'RG-Geometry'. This suggests that the 
algorithmic representation is a preferred medium for progressing in the parametric design. The protocol of student A, using a graphical algorithm editor, explicitly made and changed parameters, while in student B's and C's protocol it was difficult to distinguish between the use of parameters and rules. They used a script editor which writes the programming language to progress design.

However, the protocol of Student $\mathrm{C}$ shows that when generating a design solution or its variation, existing parameters were changed rather than the rules themselves. Making rules (RA-Rule) accounted for approximately $20 \%$ of all cognitive design activities and changing rules (RA-ChangeRule) accounted for an average of $16.3 \%$. There was some differentiation among participants regarding the coding result of 'changing rules'. Students A and C made new rules rather than changed existing ones, while Student B tended toward changing existing rules. This indicates that there are significant differences in approaches to parametric designing in relation to scripting. The third most dominantly occurring activity is the code 'SE-Geometry'.

Table 3 The percentage of coding result of each student's protocol

\begin{tabular}{|c|c|c|c|c|c|c|}
\hline \multirow{2}{*}{ Creative Level } & \multirow{2}{*}{ Subclass } & \multicolumn{3}{|c|}{ Student } & \multirow{2}{*}{ Mean } & \multirow{2}{*}{ SD } \\
\hline & & $\mathbf{A}$ & B & $\mathbf{C}$ & & \\
\hline \multirow{2}{*}{$\begin{array}{l}\text { Representation } \\
\text { (RE-Geometry) }\end{array}$} & RG-Geometry & - & 1.2 & - & 0.4 & 0.7 \\
\hline & RG-Change & - & 2.8 & 0.3 & 1.0 & 1.5 \\
\hline \multirow{6}{*}{ (RE-Algorithm) } & RA-Parameter & 5.9 & 2.8 & 0.1 & 2.9 & 2.9 \\
\hline & RA-Change Parameter & 11.2 & 0.9 & 4.6 & 5.6 & 5.2 \\
\hline & RA-Rule & 17.7 & 19.2 & 21.5 & 19.5 & 1.9 \\
\hline & RA-ChangeRule & 10.2 & 26.6 & 11.9 & 16.3 & 9.0 \\
\hline & RA-Reference & - & 2.7 & 0.2 & 1.0 & 1.5 \\
\hline & R-Generation & 0.9 & 2.8 & 12.6 & 5.4 & 6.3 \\
\hline \multirow[t]{3}{*}{ Perception } & PG-Geometry & 1.1 & 0.4 & 1.7 & 1.1 & 0.7 \\
\hline & PA-Algorithm & 2.3 & 8.2 & 3.8 & 4.8 & 3.1 \\
\hline & PA-Reference & - & 1.9 & - & 0.6 & 1.1 \\
\hline \multirow{3}{*}{$\begin{array}{l}\text { Searching for a } \\
\text { Solution } \\
\text { (Finding Idea) }\end{array}$} & SF-Initial Goal & 3.9 & 1.0 & 0.3 & \begin{tabular}{|l|}
1.7 \\
\end{tabular} & 1.9 \\
\hline & SF-Geometry SubGoal & 7.1 & 2.2 & 2.4 & 3.9 & 2.8 \\
\hline & SF-Algorithm Subgoal & 6.5 & 1.0 & 4.3 & 3.9 & 2.8 \\
\hline \multirow{4}{*}{ (Evaluation) } & SE-Geometry & 18.6 & 8.7 & 16.2 & 14.5 & 5.2 \\
\hline & SE-Parameter & 4.9 & - & - & 1.6 & 2.8 \\
\hline & \begin{tabular}{|l} 
SE-Rule \\
\end{tabular} & 6.3 & 17.0 & 19.0 & 14.1 & 6.8 \\
\hline & SE-Reference & - & - & 0.2 & 0.1 & 0.1 \\
\hline \multirow{4}{*}{ (Adopting Idea) } & SA-Geometry & 0.5 & 0.6 & 0.6 & 0.6 & 0.1 \\
\hline & SA-Parameter & 2.5 & - & 0.2 & 0.9 & 1.4 \\
\hline & SA-Rule & 0.4 & - & 0.1 & 0.2 & 0.2 \\
\hline & SA-Reference & - & - & - & 0.0 & 0.0 \\
\hline \multicolumn{2}{|l|}{ Sum } & 100 & 100 & 100 & 100 & - \\
\hline
\end{tabular}


SE-Geometry (evaluating geometries in a $3 \mathrm{D}$ view) has the highest frequency in the protocols of Students A and B, and has the second highest frequency occurring in Student C's protocols. This indicates that in parametric design environments, the design solution should be repeatedly examined using a 3D view to support conceptual design processes. Bilda and Demirkan [4] claim that it is time consuming to switch between the different visual representations that digital environments provide. In the conceptual design stage, sketching activities produce rich 2D and 3D imagery, while designers using $\mathrm{CAD}$ tools must switch to $3 \mathrm{D}$ view options. Furthermore, Students B and C's design strategies employed the writing of scripts as the main method to generate their designs, and produced many instances of the SE-Rule code (evaluating existing rules) in the verification of their algorithms. Parametric design processes produce results and generate solutions that may be unexpected and/ or complex. Consequently, what is generated during the parametric design process must be evaluated in the $3 \mathrm{D}$ view as well as in the scripting view.

The summed coverage data in each 10-min interval was visualized using the normalized coverage value (normalized $\mathrm{A}=(\mathrm{A}-$ mean) $/ \mathrm{SD})[9,34]$ to facilitate the exploration of sequential patterns. Figure 2 shows the normalized coverage of geometric and algorithmic codes over time. It enables the representation of the cognitive design activity relating to geometric and algorithmic actions in sequence and to compare the changes over time.

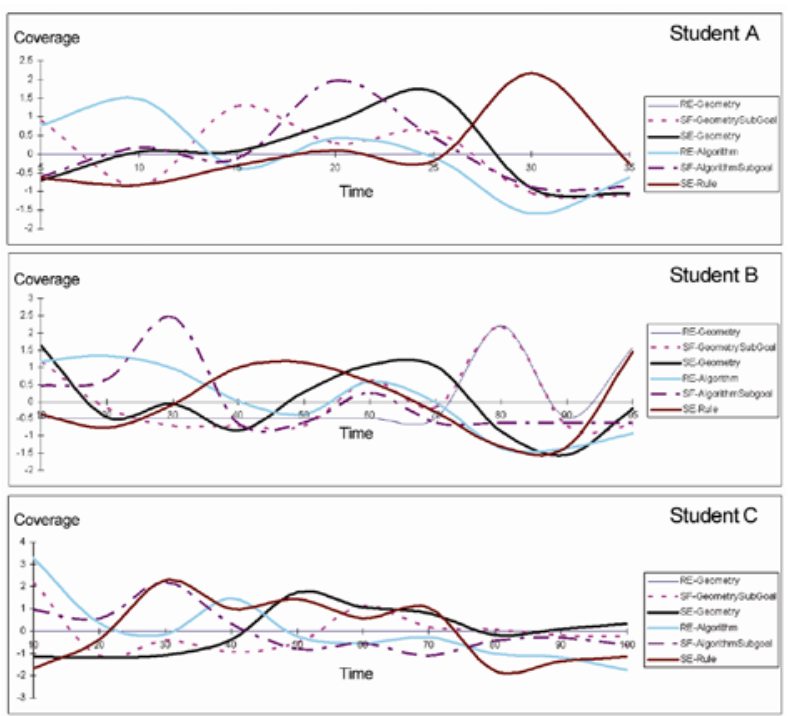

Fig 2. The normalized coverage of geometric and algorithmic activities over time 
A closer examination of Figure 2 reveals that only Student B has activities coded as 'RE (representation)-Geometry' which is 'to create or change geometries without an algorithm'. This is because Students A and $\mathrm{C}$ used algorithmic methods to achieve the manipulation of geometry. Student B has a different design strategy using both geometric and algorithmic methods to represent the design solution. This student used algorithmic methods for designing a skin pattern and a combination of geometric and algorithmic methods to model the cylindrical form of the building. In this protocol, the pattern of 'SF-Geometry Subgoal (introducing new geometric ideas extended from a previous idea)' overlaps that of 'RE-Geometry'.

The graphs in Figure 2 describe the normalized coverage of 'SEGeometry' and reveal a relatively high value in the middle of each timeframe. Whilst 'RE (representation)-Algorithm' is a dominant feature in Table 3, the normalized value of these codes decreases over time. In the case of 'SF-Algorithm Subgoal', both Students B and C utilized a script editor and have the same pattern of the normalized value. Other activities based on algorithmic methods also have a similar pattern. To solve the design problem, Students B and C introduced new algorithms to represent design ideas which were extended from a previous idea at the earlier stage of each timeframe. Activities coded as 'RA-Rule' in Student C's protocol often occurred later in the design process.

Figure 3 is intended to present the normalized coverage of the different levels of creativity (i.e., Representation, Perception and Searching for a Solution) over time and the sequential changes of these three levels of creativity. While Figure 2 illustrates the cognitive activity related to geometric and algorithmic protocols in detail, Figure 3 deals with creativity levels to provide a better understanding of the creative process.

The coding scheme consists of five subcategories within the three main creativity levels: Representation, Perception, Finding Idea, Evaluation and Adopting Idea. The last three subcategories are at the level of 'Searching for a Solution' (refer to Table 1). Figure 3 illustrates the normalized value of the different levels for each design participant. In the case of the 'Representation' level, the value decreases over time and each student produces a similar pattern. Whilst the code 'Perception' shows a relatively small amount of coverage in Table 3, Figure 3 reveals that there are different patterns within each timeframe. The 'Finding Idea' subcategory in all protocols reveals a similar pattern to the 'Adopting Idea' subcategory. We can assume that each designer's introduction of ideas follows a decision making activity. Both Students B and C who utilized a script editor produced a relatively high value of the 'Evaluation' 
subcategory in the middle of each timeframe, while Student A's increased over time.

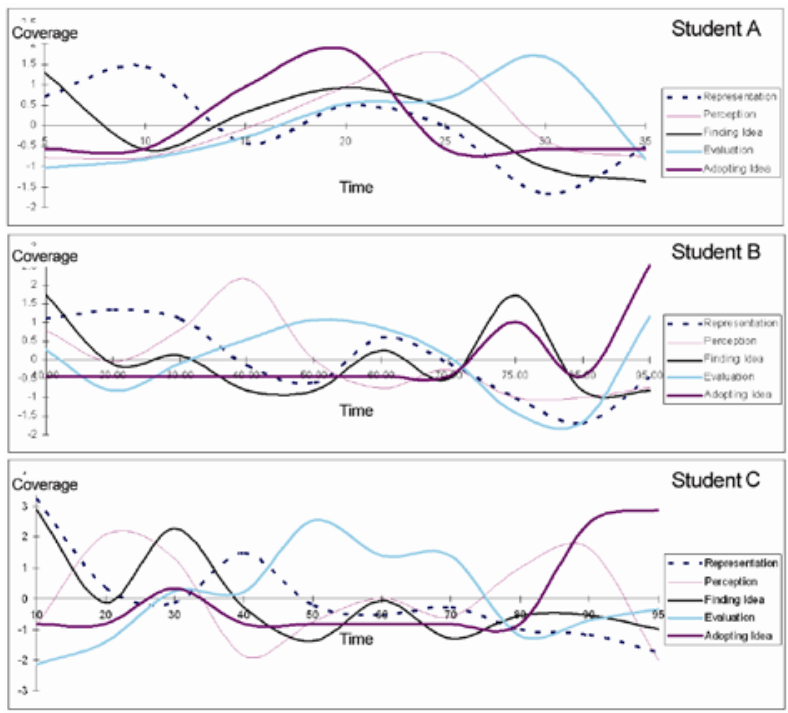

Fig 3. The normalized coverage of the creativity levels over time

These results reveal that the Design Process component of the framework is able to capture specific values of creative activities (See Table 3) and reveal a number of cognitive patterns (Figures 2 and 3) in each student's parametric design process. These results enable further analysis of the data in comparison to the rating values of the expert panel assessment.

\subsection{Product-based Evaluation of Creativity}

This section presents the implementation of the second component in the framework, namely the expert panel assessment. Figure 4 shows an overview of each student's design process relative to solutions generated. It is expected that the coding results and the design behavioral patterns will correlate with the rating values of the expert panel assessment.

\section{Assessment Experiment Procedure}

To evaluate the creativity of the design product, a panel consisting of five expert judges provided assessment of the three parametric design models produced by the students. These judges fulfilled the following criteria: 
- A tertiary degree in architecture design.

- Minimum five years of professional architectural design experience or five years of architectural design studio teaching experience.

- Familiarity with parametric design.

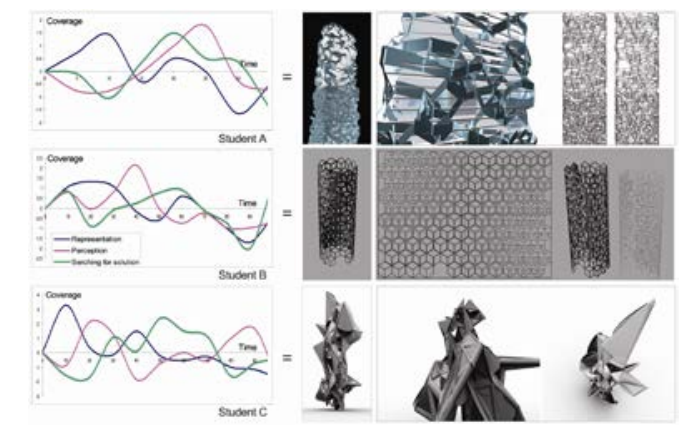

Fig 4. Students' design processes and products

Parametric design solutions were presented as a collage of images on A4 size papers so that all design products were similarly scaled. The judges had access to all images in a de-identified form. They were aware that the designs had been produced using a parametric design environment and were asked to assess designs using three evaluation forms viz.

- Assessment 1 (A1): independent non-criteria based assessment of creativity,

- Assessment 2 (A2): comparative non-criteria based assessment of creativity, and

- $\quad$ Assessment 3 (A3): criterion-based assessment of creativity using (A3i): Novelty; (A3ii): Usefulness, and (A3iii): Complexity.

Each assessment task used a seven-point Likert scale (where 1 is lowest and 7 is highest). In addition, in tasks $A 1$ and $A 2$, judges were asked to list the assessment criteria used to evaluate the level of creativity, thereby providing insights into their rationale and design focus.

\section{Results of Expert Panel Assessment}

The three students satisfied four of the five design requirements, provided in the brief. The final requirement (that the design solution be designated as a regional landmark) was included as a condition of the $A 3$ evaluation tasks, i.e., the level of novelty, usefulness, and complexity was conditional on the requirement that it reflects the characteristics of being a regional landmark. Table 4 shows the results of the judges' assessments of the three parametric design models. 
Table 4 Assessment scores of the three parametric models

\begin{tabular}{|c|c|c|c||c|c|c|}
\hline & $\begin{array}{c}\text { A1 } \\
\text { Indp. non-criteri } \\
\text { based) }\end{array}$ & $\begin{array}{c}\text { A2 } \\
\text { (Comp. non- } \\
\text { criteria based) }\end{array}$ & $\begin{array}{c}\text { A3 } \\
\text { (Combined } \\
\text { criteria) }\end{array}$ & $\begin{array}{c}\text { A3i } \\
\text { (Novelty } \\
\text { Criterion) }\end{array}$ & $\begin{array}{c}\text { A3ii } \\
\text { (Usefulness } \\
\text { Criterion) }\end{array}$ & $\begin{array}{c}\text { A3iii } \\
\text { (Complexity } \\
\text { Criterion) }\end{array}$ \\
\hline Student A & 5.4 & - & 4.8 & 4.6 & 5.2 & 4.6 \\
\hline Student B & 4.8 & Lowest & 3.6 & 2.8 & 3.4 & 4.6 \\
\hline Student C & 6.4 & Highest & 6.0 & 6.4 & 5.2 & 6.4 \\
\hline Mean & 5.5 & - & 4.8 & 4.6 & 4.6 & 5.2 \\
\hline SD & 0.8 & - & 1.2 & 1.8 & 1.0 & 1.0 \\
\hline
\end{tabular}

The values shown in Table 4 are the average grades of the five judges' assessments. The final column shows the design solution's (i.e., student's) average grade received for each criterion. It also shows that the average values for the non-criteria based assessment are slightly higher than the average values in the criterion based assessment for each of the three criteria assessed.

The results consistently show that the level of creativity of Student C's model is assessed by the expert panel as being the highest across all evaluations, with the exception of task A3ii, where Student B's model has an equivalent score for the level of usefulness as a regional landmark. This is confirmed in the average of all assessment tasks (the final column).

For tasks $A 1$ and $A 2$ each panel member listed a range of criteria used to evaluate the level of creativity in these non-criteria based evaluations. Responses were categorized as shown in Table 5 and revealed a number of variations and similarities in the criteria used. The two most common responses, highlighted below, included 'Technical complexity' and 'Aesthetic attractiveness'.

Table 5 Individual criteria used by each judge for assessing creativity

\begin{tabular}{|l|l|l|l|l|l|}
\hline & Judge & $\begin{array}{l}\text { Judge } \\
\mathbf{1}\end{array}$ & $\begin{array}{l}\text { Judge } \\
\mathbf{1}\end{array}$ & $\begin{array}{l}\text { Judge } \\
\mathbf{4}\end{array}$ & $\begin{array}{l}\text { Judge } \\
\mathbf{5}\end{array}$ \\
\hline Novelty & & $\checkmark$ & & $\checkmark$ & $\checkmark$ \\
\hline Functionality quality & & $\checkmark$ & $\checkmark$ & & \\
\hline Technical complexity & $\checkmark$ & $\checkmark$ & & $\checkmark$ & $\checkmark$ \\
\hline Technical integrity & & & $\checkmark$ & $\checkmark$ & $\checkmark$ \\
\hline Integrative capacity & & & & & $\checkmark$ \\
\hline Aesthetic complexity & $\checkmark$ & & $\checkmark$ & & \\
\hline Aesthetic attractiveness & $\checkmark$ & $\checkmark$ & $\checkmark$ & $\checkmark$ & \\
\hline
\end{tabular}




\subsection{The Mapping of Process and Product Creativity Evaluations}

Mapping the cognitive activities of the design process and the expert's rating of the design product was performed by correlating the following sets of data: coding results (Table 3), non-criteria-based creativity and criteria-based creativity evaluation scores (Table 4). Some visualized patterns in Figure 2 and 3 were also used to inform the mapping process. In order to effectively deal with different types of data and values in Tables 3 and 4, the normalized values for each code and rating were calculated and presented relative to each student's cognitive activities. Various types of comparisons have been produced to explore the implication of the results. They are shown in Figure 5 and Table 6.

Possible patterns emerging from the comparisons are listed in the lefthand-side column of Table 6. For example, 'A $>B>C$ ' means that Student A's is higher than Student B's, and Student B's is higher than Student C's; ' $A>B \approx C$ ' means that Student A's is the highest, and Student B's and Student C's are similarly lower; ' $A<B<C$ ' means that Student $A$ 's is the lowest, and Student C's is the highest; ' $A<B>C$ ' means that Student B's is the highest, and Student A's and Student C's are similarly lower; ' $A>B<C$ ' means that Student B's is the lowest, and Student A's and Student C's value are similarly higher. The system allows us to easily relate the coding results of the design process to the rating results of the design product.

Due to the limited sample size of the pilot study, we selected those patterns that are consistent with the expert panel's results for a surface discussion on the correlation between the parametric design process and product. The fifth process pattern $(\mathrm{A}>\mathrm{B}<\mathrm{C})$ in Table 6 is consistent with the results, i.e. activities 'PG-Geometry', 'SF-Algorithm Subgoal', 'SEGeometry', and 'RA-Change Parameter' are revealed as important aspects for supporting creativity in parametric design. More detailed implication analysis and other correlation analyses will be explored and generalized in future work.

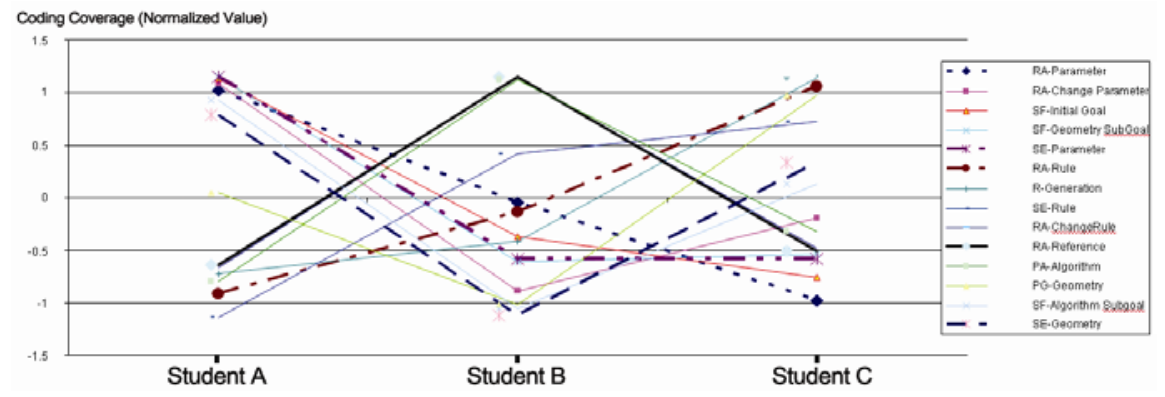

Fig 5. Comparison between the three students based on the normalized values of each code 
Table 6 Main patterns emerged in the comparison between the three students

\begin{tabular}{|c|c|c|c|c|}
\hline Patterns & Code & Student A & Student B & Student C \\
\hline \multicolumn{5}{|c|}{ Process (Normalized values for each code) } \\
\hline \multirow{2}{*}{$\mathrm{A}>\mathrm{B}>\mathrm{C}$} & RA-Parameter & 1.02 & -0.05 & -0.98 \\
\hline & SF-Initial Goal & 1.13 & -0.37 & -0.76 \\
\hline \multirow{2}{*}{$\mathrm{A}>\mathrm{B} \approx \mathrm{C}$} & SF-Geometry SubGoal & 1.15 & -0.61 & -0.54 \\
\hline & SE-Parameter & 1.15 & -0.58 & -0.58 \\
\hline \multirow{3}{*}{$\mathrm{A}<\mathrm{B}<\mathrm{C}$} & RA-Rule & -0.92 & -0.14 & 1.06 \\
\hline & R-Generation & -0.72 & -0.42 & 1.14 \\
\hline & SE-Rule & -1.14 & 0.42 & 0.72 \\
\hline \multirow{3}{*}{$\mathrm{A}<\mathrm{B}>\mathrm{C}$} & RA-ChangeRule & -0.67 & 1.15 & -0.48 \\
\hline & RA-Reference & -0.64 & 1.15 & -0.51 \\
\hline & PA-Algorithm & -0.80 & 1.12 & -0.32 \\
\hline \multirow{4}{*}{$\underline{\mathrm{A}>\mathrm{B}<\mathrm{C}}$} & PG-Geometry & 0.05 & -1.02 & 0.97 \\
\hline & SF-Algorithm Subgoal & 0.93 & -1.06 & 0.13 \\
\hline & SE-Geometry & 0.79 & -1.12 & 0.33 \\
\hline & RA-Change Parameter & 1.08 & -0.89 & -0.19 \\
\hline \multicolumn{5}{|c|}{ Product (Assessment scores) } \\
\hline \multirow{3}{*}{$\begin{array}{l}\frac{A>B<C}{\&} \\
A<C\end{array}$} & A1-Indp. non-criteria based & 5.40 & 4.80 & 6.40 \\
\hline & A3-Combined Criteria & 4.80 & 3.60 & 6.00 \\
\hline & Mean & 4.92 & 3.84 & 6.08 \\
\hline
\end{tabular}

\section{Discussion and Conclusion}

This paper aims to explore and evaluate creativity in parametric design. The framework for this evaluation presented here shows that the underlying cognitive activities of the creative process can be described by the encoding schema and identified via protocol analysis. It further demonstrates that parametric design outputs can be formally evaluated using CAT. A mapping process through correlation analysis was used to identify the relationships between designers' cognitive patterns during the design process and the evaluation scores of the product. The preliminary evidence shows that the combined process-product approach is capable of revealing the conditions that potentially enhance creativity in parametric design. Overall, based on the correlation analysis (Table 6), this two-part framework performs well, revealing insights into the creative process and product in parametric design.

As a pilot study, the research reported here comes with limitations in the generalization of results. Whilst the authors acknowledge the small sample size, the results show some unique characteristics of creativity in parametric design. First of all, the paper highlights that the coverage of 
'Searching for a Solution' is dominant and changing existing parameters or rules, as an algorithmic activity, is related to the potential production of creative outcomes. 'RA-Change Parameter' is an important activity for supporting creativity as shown in Table 6 . In parametric design, rules are often regarded as graphical programming tools that carry constraints, while parameters are related to generating alternatives and reconstructing the design process. This implies that parametric design provide tools that can better support divergent thinking as well as providing reconstructing [10, 13] and regulating [8] processes, which enhance creativity.

'SE-Geometry' was also revealed as another dominant activity in parametric design. It can be assumed that more frequent evaluation activities potentially refine the design solution. The results of correlation analysis also support 'SE-Geometry' as potentially one of the most essential activities supporting creativity in parametric design. Furthermore, as shown in Figures 2 and 3, different cognitive patterns can be indirectly related to creativity. For example, Students B and C who use a script editor have a similar pattern as indicated by the normalized values revealed in these figures. Student $\mathrm{C}$ who receives higher scores from judges shows a clear sequential patter of 'Finding Idea', 'Evaluation', and 'Adopting Idea'. These activities have previously been identified as common processes in a number of models of creative processes [26]. This shows that parametric design has the potential to support a more flexible and intuitive design process as well as a creative mode of designing. The criteria used by the panel to assess the product (Table 5) still show the duality of both technical fitness and aesthetic attractiveness [35]. The reciprocal aspect of parametric design may be one of the most interesting features in terms of creativity.

In summary, the pilot study presents promising results demonstrating the effectiveness of the framework in evaluating parametric design creativity. Such a conceptual framework has not previously been available. The paper demonstrates and verifies the framework using the two-part pilot study. Using protocol analysis, the expert panel assessment and a correlation analysis by mapping the two, illustrate the framework's potential to investigate and contribute to our understanding of the nature of creativity in parametric design. However challenges remain; the key challenge lies with further testing of the framework using larger sample sizes in both the parametric design and the expert panel assessments. Future work therefore includes (1) verification of subscale criteria in expert panel assessment (Table 2), which have not been tested exhaustively by this pilot study; (2) further investigation of the correlation results (Table 6), which are not fully explored here due to the limited 
scope of this paper; and (3) a larger study to generalize results of both evaluation methods.

\section{References}

1. Schumacher P, (2009) Parametricism: A New Global Style for Architecture and Urban Design. Architectural Design 79: 14-23

2. Lee JH, Gu N, Sherratt S (2011) Developing a framework for evaluating creativity in parametric design. In: From principles to practice in architectural science - ANZAScA2011. The University of Sydney, Abstracts Book, p 17

3. Casakin H, Kreitler S, (2011) The cognitive profile of creativity in design. Thinking Skills and Creativity 6: 159-168

4. Bilda Z, Demirkan H, (2003) An insight on designers' sketching activities in traditional versus digital media. Design Studies 24: 27-50

5. Hasirci D, Demirkan H, (2007) Understanding the effects of cognition in creative decision making: A creativity model for enhancing the design studio process. Creativity Research Journal 19: 259-271

6. Chai K-H, Xiao X, (2012) Understanding design research: A bibliometric analysis of Design Studies (1996-2010). Design Studies 33: 24-43

7. Benami O, Jin Y (2002) Creative stimulation in conceptual design. In: Proceedings of the ASME Design Engineering Technical Conference, pp. 251-263

8. Akin O, Moustapha H, (2004) Strategic use of representation in architectural massing. Design Studies 25: 31-50

9. Kim JE, Bouchard C, Omhover JF, Aoussat A, (2010) Towards a model of how designers mentally categorise design information. CIRP Journal of Manufacturing Science and Technology 3: 218-226

10. Verstijnen IM, van Leeuwen C, Goldschmidt G, Hamel R, Hennessey JM, (1998) Sketching and creative discovery. Design Studies 19: 519-546

11. Ibrahim R, Rahimian FP, (2010) Comparison of CAD and manual sketching tools for teaching architectural design. AutoCon 19: 978-987

12. Liu Y-T, (2000) Creativity or novelty? Design Studies 21: 261-276

13. Madkour Y, Neumann O, Erhan H, (2009) Programmatic Formation: Practical Applications of Parametric Design. International Journal of Architectural Computing 7: 587-604

14. Aish R, Woodbury R (2005) Multi-level Interaction in Parametric Design. In: Butz A, Fisher B, Krüger A, Olivier P (eds) Smart Graphics. Springer Berlin / Heidelberg, pp. 924-924

15. Iordanova I, Tidafi T, Guité M, Paoli GD, Lachapelle J (2009) Parametric methods of exploration and creativity during architectural design: A Case study in the design studio. In: Tidafi T, Dorta T (eds) Joining Languages, Cultures and Visions: CAADFutures 2009, pp. 423-439

16. Salim F, Burry J (2010) Software Openness: Evaluating Parameters of Parametric Modeling Tools to Support Creativity and Multidisciplinary 
Design Integration. In: Computational Science and Its Applications - ICCSA 2010. Springer Berlin / Heidelberg, pp. 483-497

17. Chusllp P, Jin V (2004) Cognitive modeling of iteration in conceptual design in the Proceedings of the ASME Design Engineering Technical Conference, pp. 473-485

18. Rhodes M, (1961) An Analysis of Creativity. The Phi Delta Kappan 42: 305310

19. Simonton DK, (2003) Scientific creativity as constrained stochastic behavior: the integration of product, person, and process perspectives. Psychol Bull 129: 475-494

20. Amabile TM, (1983) The social psychology of creativity: A componential conceptualization. Journal of Personality and Social Psychology 45: 357-376

21. Akin O (1986) Psychology of Architectural Design. Pion, London

22. Kim MJ, Maher ML, (2008) The Impact of Tangible User Interfaces on Designers' Spatial Cognition. Human Computer Interaction 23: 101 - 137

23. Suwa M, Purcell T, Gero J, (1998) Macroscopic analysis of design processes based on a scheme for coding designers' cognitive actions. Design Studies 19: 455-483

24. Gero JS, Neill TM, (1998) An approach to the analysis of design protocols. Design Studies 19: 21-61

25. Coley F, Houseman O, Roy R, (2007) An introduction to capturing and understanding the cognitive behaviour of design engineers. Journal of Engineering Design 18: 311-325

26. Hayes JR (1989) Cognitive processes in Creativity. In: Glover JA, Ronning RR, Reynolds CR (eds) Handbook of creativity Perspectives on individual differences. New York, NY, US: Plenum Press, pp. 135-145

27. Suwa M, Tversky B, (1997) What do architects and students perceive in their design sketches? A protocol analysis. Design Studies 18: 385-403

28. Amabile TM (1983) The social psychology of creativity. New York: SpringerVerlag

29. Christiaans HHCM, (2002) Creativity as a Design Criterion. Creativity Research Journal 14: 41 - 54

30. Baer J, Mckool SS, (2009) Assessing Creativity Using the Consensual Assessment Technique. Creativity Research Journal: 1-13

31. Wallas G (1926) The Art of Thought. New York: Harcourt Brace

32. Hennessey B, Amabile T (1999) Consensual assessment. In: Runco M, Pritzker S (eds) Encyclopedia of creativity. San Diego, CA: Academic Press, pp. 346-359

33. Besemer SP, O'Quin K (1987) Creative product analysis:Testing a model by developing a judging instrument. In: Isaksen SG (ed) Frontiers of creativity research: Beyond the basics. Buffalo, NY: Bearly Limited, pp. 341-357

34. Bilda Z, Gero JS, (2007) The impact of working memory limitations on the design process during conceptualization. Design Studies 28: 343-367

35. Goldschmidt G (1992) Criteria for design evaluation: a process-oriented paradigm. In: Kalay YE (ed) Evaluating and predicting design performance. Wiley Interscience, New York pp. 67-79 